\title{
COMPARATIVE STUDY ON EYE GAZE ESTIMATION IN VISIBLE AND IR SPECTRUM
}

\author{
Susmitha Mohan and Manoj Phirke
}

Imaging and Robotics Lab, HCL Technologies, Bangalore, India

\begin{abstract}
Eye gaze estimation aims to find the point of gaze which is basically," where we look". Estimating the gaze point plays an important role in many applications with varying usage. Gaze estimation is used in automotive industry to ensure safety. In the field of retail shopping and online marketing gaze estimation is used to analyse the consumer's interest and focus. Gaze estimation is also used for psychological tests and in healthcare for diagnosing some of the neurological disorders. This also has a significant role to play in the field to entertainment. There are multiple ways by which eye gaze estimation can be done. This paper is about a comparative study done on two of the popular methods for gaze estimation using eye features. An infra-red camera is used to capture data for this study. Method 1 tracks corneal reflection centre w.r.t the pupil centre and method 2 tracks the pupil centre w.r.t the eye centre to estimate gaze. There are advantages and disadvantages with both the methods which has been looked into. Choosing the right method for gaze estimation hence depends on the type of application, precision required and many other factors including environmental conditions. This paper can act as a reference for researchers working in the same field
\end{abstract}

\section{KEYWORDS}

eye gaze, pupil, iris, cornea, corneal reflection, polynomial curve fitting, infra-red.

\section{INTRODUCTION}

Eye gaze estimation tells a lot about the attention and the focus of the person. This information is useful in various ways in various fields and there are different ways by which gaze estimation is performed in accordance with the performance requirement. It is important for the web designers to understand where people gaze most on the screen and where not. This information helps them to place the right buttons at the right place. In healthcare the information about eye gaze variation helps to diagnose some of the neurological disorders and some of the developmental disorders like autism. In automobile industry gaze estimation of the driver helps to find out if he is attentive enough on the road or not. Gaze estimation is also used in the field of augmented or virtual reality. Vision aided applications also make use of eye gaze estimation to help the user.

There are different ways by which gaze estimation can be done. Some of the methods uses wearable gadgets whereas some doesn't. Analysing the movement of eye features is the underlying principle in all of the gaze estimation systems. Analysing the movement of pupil w.r.t the eye and the movement of corneal reflection w.r.t the pupil has gained a lot of attraction because of its simplicity and easiness. This movement of the eye features has been interpreted mathematically in various ways. Some of the methods like polynomial fitting and Gaussian fitting are widely used. 
With the increasing popularity and usability of gaze estimation, the accuracy and efficiency of gaze estimation is also important. The amount of accuracy and efficiency required can be different from one application to another application. Also, the minimum gaze angle to be measured varies with the kind of application it is used for. For example, in the case of a graphical user interface (GUI) developer the gaze angle measurement has to be more accurate when compared to the gaze estimation of a driver. Since, for a driver it may not be so important to understand precisely at which point on the road or outside environment he is gazing at. Whereas for a GUI developer it is important to understand precisely at which point on the screen the person is looking at. The type of environment and the lighting also plays a significant role in the selection of sensor and method for gaze estimation. The lighting variation inside a car can be large when compared to an indoor environment and hence the technology suiting the GUI developer for gaze estimation might not fit for driver gaze estimation. In case of person wearing spectacles or goggles gaze estimation is challenging and sometimes impossible when the eyes are fully occluded. For accurate estimation of eye gaze and for gaze estimation under challenging scenarios more sophisticated devices and techniques are used.

There is intense research happening over decades to improve the eye gaze estimation performance. Recent trends and developments happening in gaze estimation is present in [3], [4], [5]. In [1] visible light is used to obtain the corneal reflection instead of IR lighting. An efficient method of using geometric transforms in homography normalization (HN) method when corneal reflections are lesser than 4 in number is present in [6]. The possibility of accurately detecting and tracking human gaze based on the head pose information extracted by an RGB-D device is present [7]. A mapping function based on artificial neural networks is used in [8]. How well Gaussian process adapt to the non-linearity in eye movement over polynomial regression is presented in [2]. In [9] Fourier descriptors are used to describe the appearance-based features of eyes compactly. Shape and intensity based deformable eye pupil-centre detection and movement decision algorithms is present in [10]. An effective way of estimating eye gaze from the elliptical features of one iris is described in [11]. An investigation on the effect of gaze position on pupil size estimation by three common eye-tracking systems is presented in [12]. A low-cost system which uses web camera and open source Computer Vision Library Open CV is proposed in [13]. In [14] and [15] review of different eye tracking systems for diagnostic interpretation is presented. A review of eye gaze estimation techniques and applications for consumer products, which has progressed in diverse ways over the past two decades is presented in [16]. In [17] a new hardware friendly, convolutional neural network (CNN) model with minimal computational requirements is introduced and assessed for efficient appearance-based gaze estimation.

In this paper two of the popular gaze estimation methods are compared. Method 1 will be referred to as 'corneal reflection-based gaze estimation' and method 2 will be referred to as 'pupil-based gaze estimation' in the rest of the paper. Pupil based gaze estimation doesn't need an infra-red (IR) camera for gaze estimation whereas corneal reflection-based method needs an IR camera to function. But for comparison purpose data captured from an IR camera is used for both pupil based and corneal reflection-based analysis as both the eye features are visible in IR data. This paper is hence a comparison of what can be achieved with an IR camera and without an IR camera. Both the methods have advantages and disadvantages which will be discussed in detail in further sections

\section{Eye Features AND CORNEAL REFLECTION}

For pupil-based gaze estimation method features of the eye like pupil centre and eye corner points are used for gaze estimation. For corneal reflection-based method features of the eye like 
iris, pupil centre and the bright spot formed by the IR illuminators on the eye are used for gaze estimation. Figure 1 shows the eye features and corneal reflection.

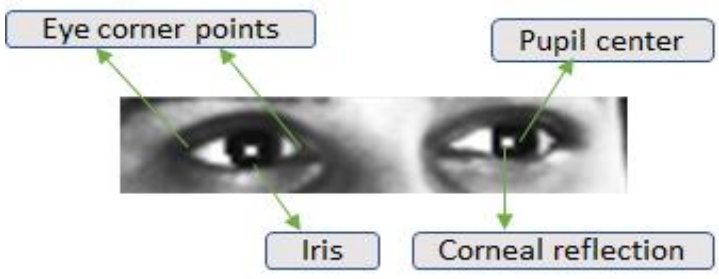

Figure 1. Eye features and corneal reflection.

\section{Camera Setup and Data Capture}

An IR camera is placed in between the person and a fixed pattern. The fixed pattern has markings in both vertical and horizontal directions to gaze at. To compare the pupil movement and corneal reflection movement images of eyes are captured using the IR camera looking at the frontal face of the person. The camera set up consists of an IR lens, set of IR illuminators that emits IR radiation within a specific bandwidth and an IR filter that allows only the radiation falling in this specific bandwidth to pass through. This set up minimizes image quality variations caused by surrounding illumination changes. Figure 2 shows the fixed pattern and the camera setup used for capturing data.

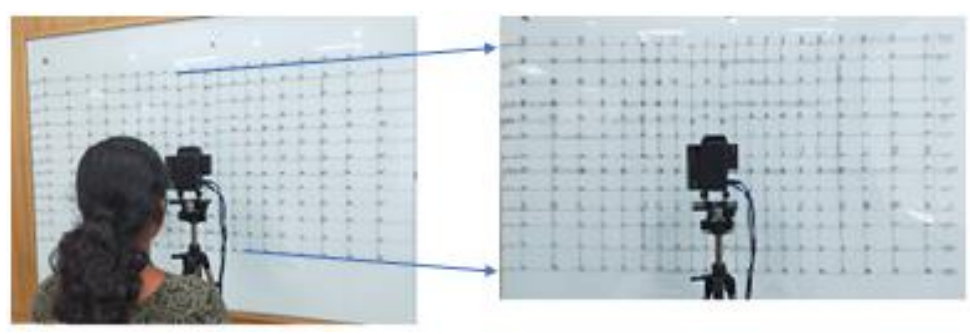

Figure 2. Fixed pattern to gaze at Images of eyes at different gaze angle are captured using the IR camera.

When the gaze changes from one point to the next point on the fixed pattern only the eyes are moved without any head movement. Sets of images are captured with eye gaze varying in horizontal direction from left to right with an offset of 5 degree and the vertical gaze angle kept constant. On successful completion of one set of horizontal data capture the vertical gaze is changed to 5 degree up or down and next set of horizontal eye gaze data is captured. The maximum variation in eye gaze in horizontal direction is from -45 degree to +45 degree and in the vertical direction is from -30 degree to +30 degree using the pattern shown in figure 2 .

\section{GaZe Estimation MethodS}

Two of the commonly used gaze estimation methods are chosen and compared in this paper. In corneal reflection-based method the movement of corneal reflection w.r.t the pupil centre is analysed and in pupil-based method the movement of pupil centre w.r.t the eye centre is analysed. Corneal reflection formation requires an IR camera along with IR illuminators, whereas for pupil movement analysis any normal camera would suffice. But for accurate and efficient comparison data set used for both the methods are captured using an IR camera as the images includes all the features required for both the methods. This study can also be considered as a comparative study of what can be achieved with an IR camera and what can be achieved with a 
normal camera. From the face images captured using the IR camera, eye region is detected and used for both the methods. Any state-of-the art method can be used to detect eye region, eye features and corneal reflection point. Eye features include corner points of eye, boundary of iris, pupil and pupil centre.

\subsection{Corneal reflection-based gaze estimation}

Near-infrared light directed towards the eyes forms bright reflections on the cornea called corneal reflection. As there are two sets of illuminators placed on either side of the lens, two bright spots are formed on the cornea. Centre of these two bright spots is referred to as corneal reflection centre in the whole paper and this spot is considered for the analysis. With the changes in eye gaze the corneal reflection centre moves around the pupil in some specific pattern. This specific pattern helps to estimate gaze angle. To find the relation between corneal reflection movement and gaze angle, measurements shown in (1), (2) and (3) are used.

$$
\begin{aligned}
& \mathrm{x}=\mid \text { PupilCenter_x }- \text { CornealReflectionCenter_x } \mid \\
& \mathrm{y}=\mid \text { PupilCenter_y }- \text { CornealReflectionCenter_y } \mid \\
& \mathrm{R}=\text { Radius of iris }
\end{aligned}
$$

Figure 3 has $x, y$ and $R$ marked

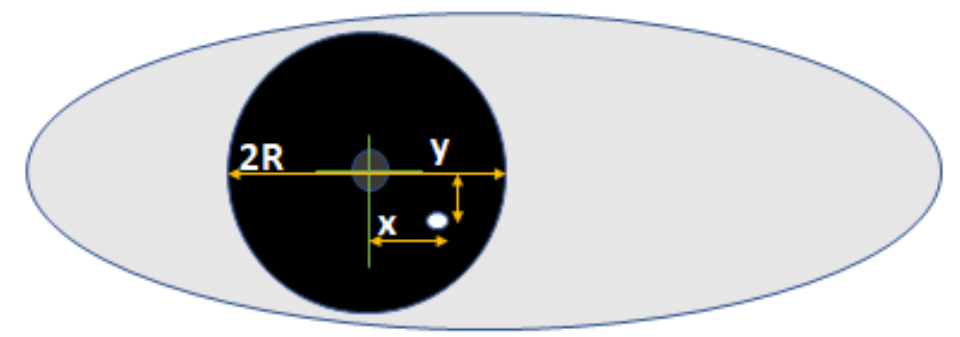

Figure 3. Measurements for gaze estimation from corneal reflection

\subsubsection{Horizontal gaze estimation using corneal reflection}

To estimate horizontal gaze angle from corneal reflection eye gaze is shifted only in the horizontal direction from left to right and vertical gaze angle kept constant. It is observed that when camera is aligned with the centre of face and the gaze is at the centre of the fixed pattern then the corneal reflection centre overlaps with the centre of pupil. When the gaze shifts towards left from the centre of the pattern then the corneal reflection moves towards right from the pupil centre. And when the gaze shifts towards right from the centre of the pattern then the corneal reflection moves towards left from the pupil centre. The corneal reflection moves farther from the pupil centre when the gaze shifts more towards left or right from the centre of the pattern. But this relation is not linear. To find the relation between corneal reflection position and horizontal gaze angle ' $\mathrm{x} / \mathrm{R}$ ' is calculated for different horizontal gaze angles with vertical gaze angle kept constant. Instead of ' $x$ ' and ' $y$ ', ' $x / R$ ' and ' $y / R$ is used in the analysis to remove the dependency on image resolution and distance from the camera. The values obtained for ' $\mathrm{x} / \mathrm{R}$ ' for varying horizontal angles are as shown in Figure 4.

It is clear from the values that the relation is not linear as the difference between subsequent values is not constant. To find the non-linear relation connecting corneal reflection position with eye gaze angle, ' $\mathrm{x} / \mathrm{R}$ ' is plotted against horizontal eye gaze and different curve fittings are tried on these calibration points. 


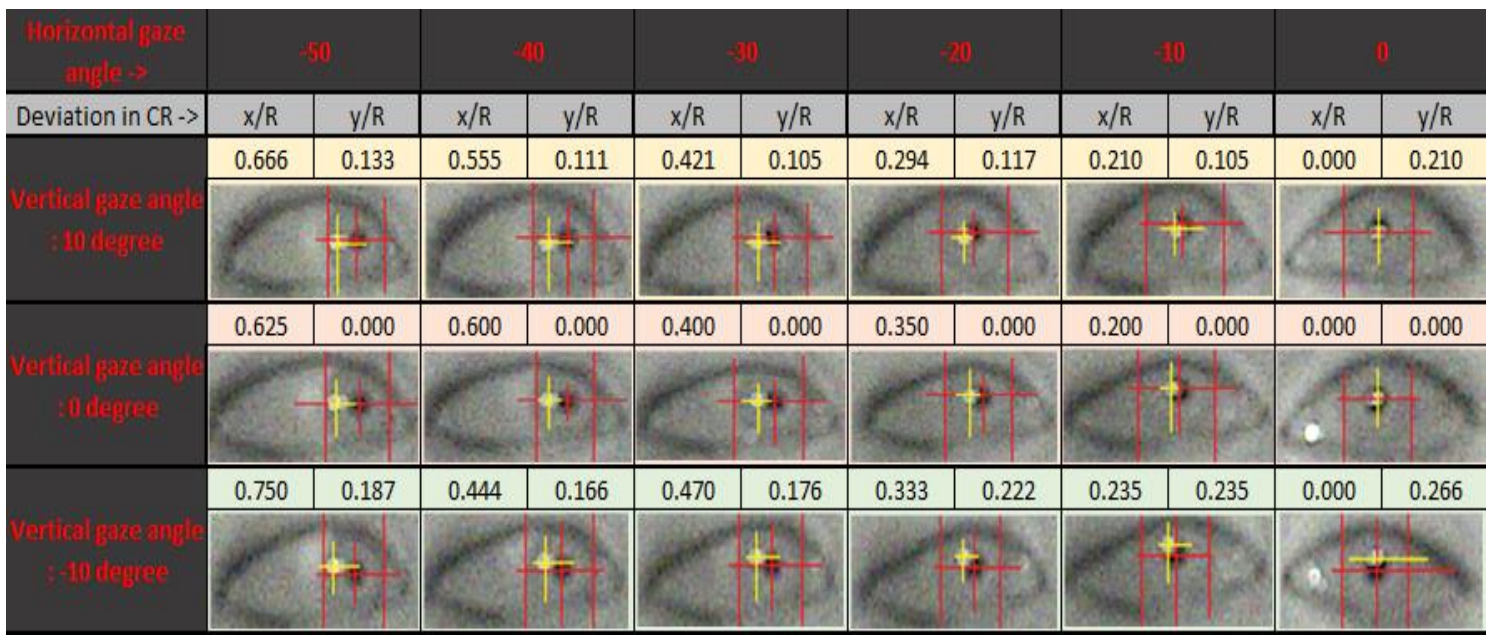

Figure 4. $\mathrm{x} / \mathrm{R}$ values for different gaze angles

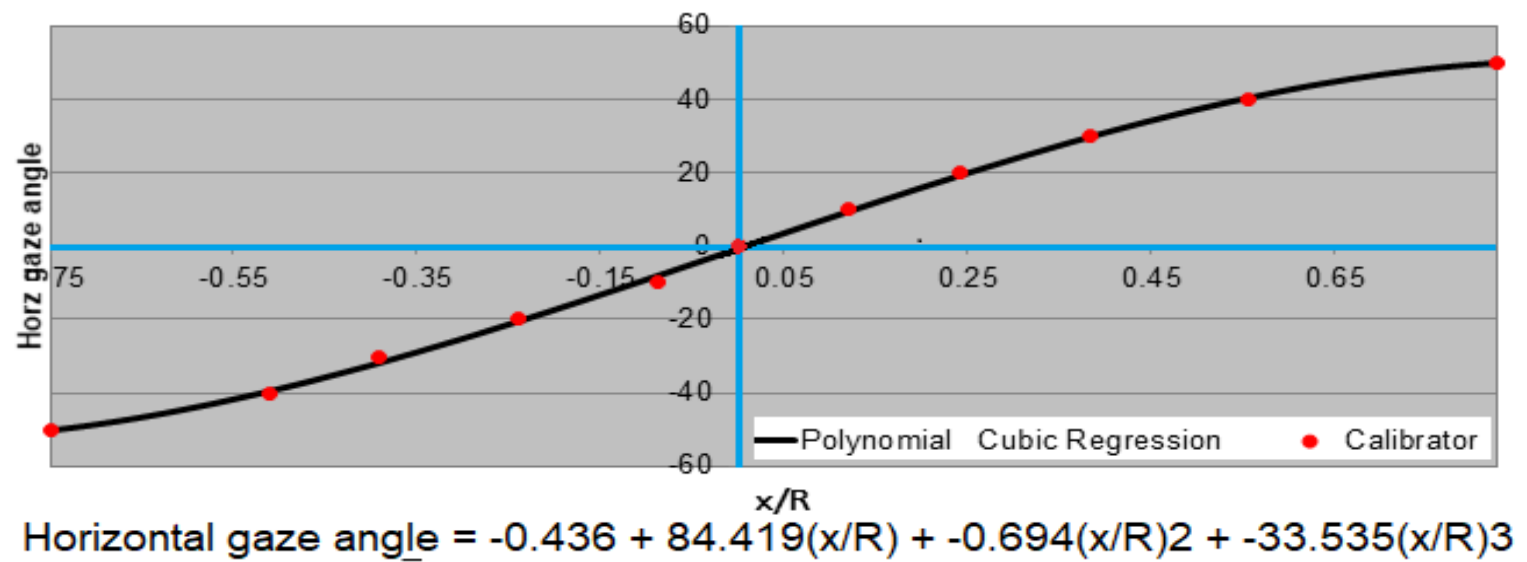

Figure 5. $\mathrm{x} / \mathrm{R}$ vs gaze angle plot

From figure 5 it can be observed that a third order polynomial is fitting the calibration points effectively. For easy comparative analysis polynomial regression is preferred in this paper over Gaussian or other methods. As the relation between horizontal gaze angle and corneal reflection position is established as a polynomial function, it is possible to calculate the gaze angle from the iris dimensions, pupil and corneal reflection centre position.

HorzGazeAngle $=-0.436+84.419(\mathrm{x} / \mathrm{R})+-0.694(\mathrm{x} / \mathrm{R}) 2+-33.535(\mathrm{x} / \mathrm{R}) 3$

For a given person the relation represented in 4 is true for varying distance between camera and eyes. This relation is also true at varying vertical eye gaze angle. Figure 6 has 'x/R' values at different vertical gaze angle and horizontal gaze angle kept constant (30 degree). 


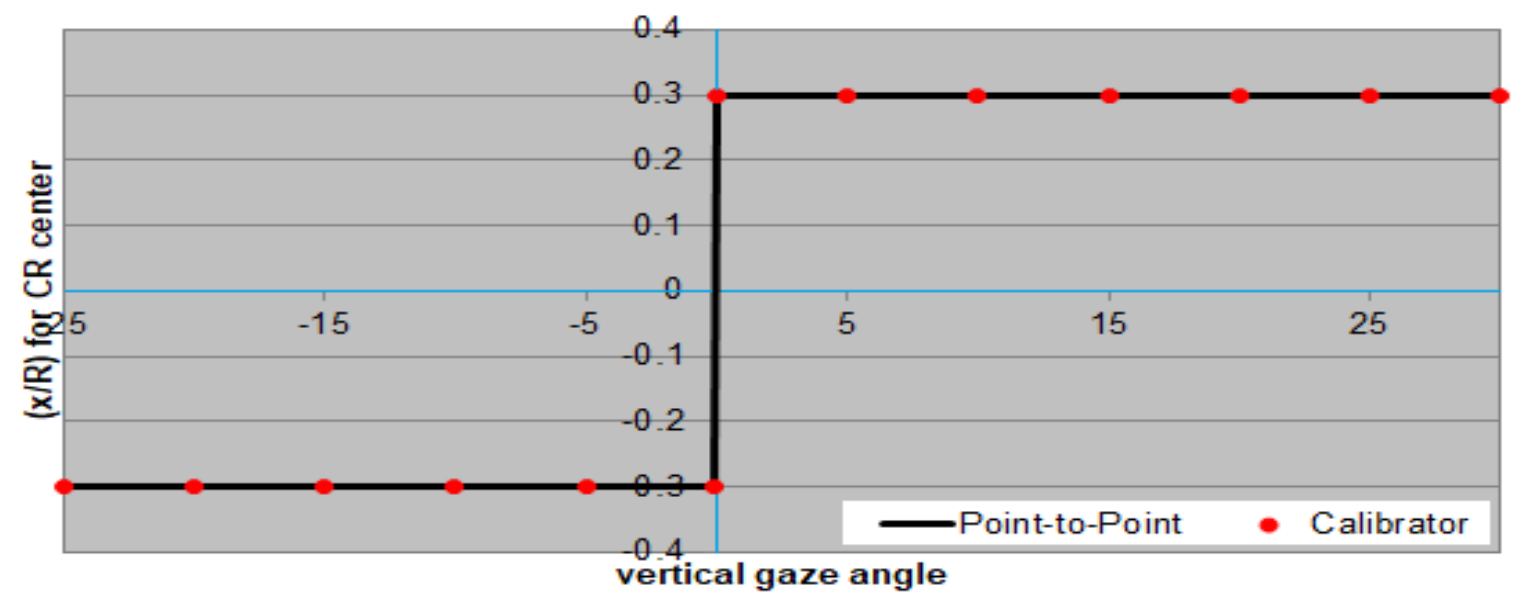

Figure 6. $x / R$ at varying vertical gaze angle

As observed in figure $6^{\prime} \mathrm{x} / \mathrm{R}^{\prime}$ is a constant at different vertical gaze angle with horizontal gaze angle kept constant.

When camera is not aligned with the centre of both eyes, then there is a shift in the polynomial curve from zero. The curve plotted in Figure 7 is with the camera shifted towards right by 10 degrees.

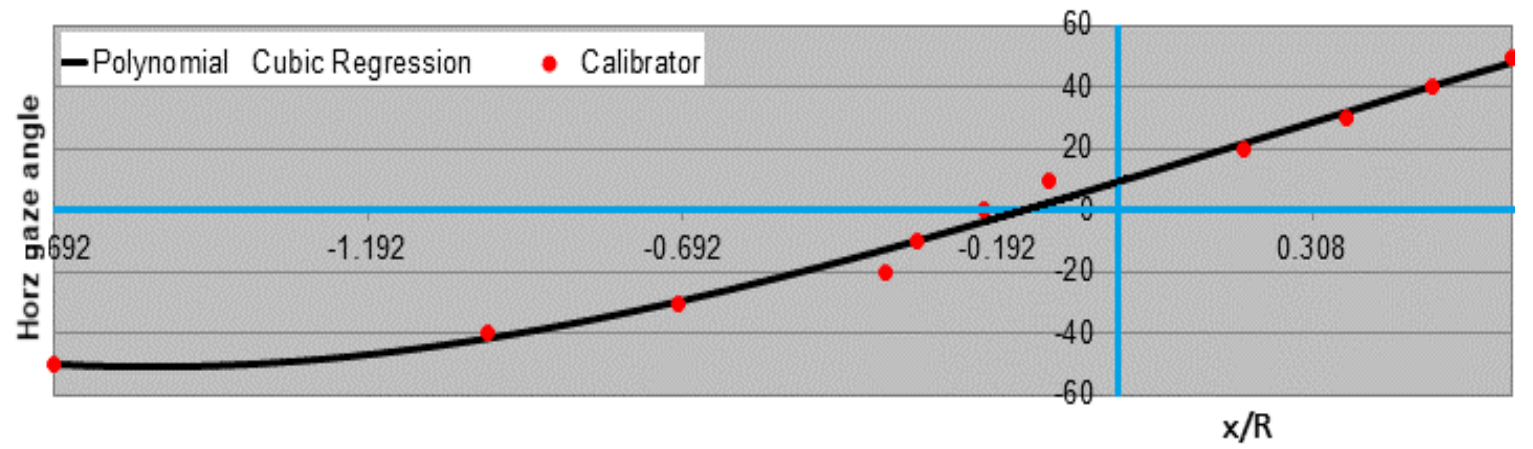

\section{Horizontal gaze angle $=9.54+62.08(x / R)+4.21(x / R) 2+-6.93(x / R) 3$}

Figure 7. Shift in curve fitting with the shift in camera alignment

It is important that any gaze estimation method should be generic enough to work on multiple people. To check how generic is curve fitting method for gaze estimation, eye images with horizontal gaze varying from -50 to +50 degree and vertical gaze angle kept as zero is collected from different people and a third order polynomial curve is fitted on these calibration points. Figure 8 has values from 3 different people marked in three different colours. The error in curve fitting is calculated and is found to be minimal. Error values of each coefficient of curve fitting has values as shown in table $\mathrm{I}$. 


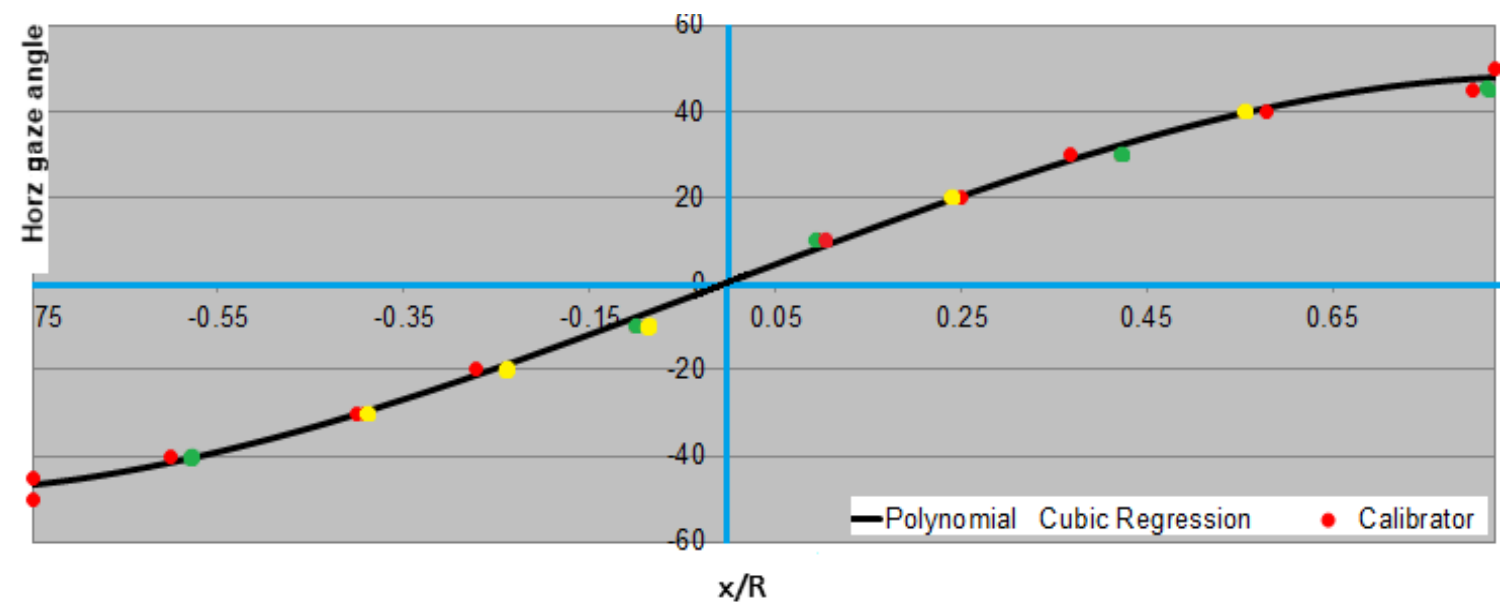

Horizontal gaze angle $=0.26+81.85(x / R)+-0.79(x / R) 2+-34.82(x / R) 3$

Figure 8. $\mathrm{x} / \mathrm{R}$ values from 3 different people

Table 1: Error values of polynomial coefficients

\begin{tabular}{|c|c|c|}
\hline Coeff & Value & Error \\
\hline $\mathrm{a}$ & 0.26 & 0.638 \\
\hline $\mathrm{b}$ & 81.85 & 2.192 \\
\hline $\mathrm{c}$ & -0.79 & 2.024 \\
\hline $\mathrm{d}$ & -34.82 & 4.576 \\
\hline
\end{tabular}

The polynomial curve in figure 8 is used to estimate gaze for person 1,2 and 3 and the error in gaze estimation is obtained as $1.33 \mathrm{deg}, 1.61 \mathrm{deg}$ and $1.11 \mathrm{deg} \mathrm{respectively.} \mathrm{When} \mathrm{'} \mathrm{y} / \mathrm{R}$ ' is plotted against varying horizontal eye gaze angles (with vertical gaze angle kept constant) then the plot as shown in Figure 9 is obtained. As observed from Figure 9 ' $y / R$ ' is constant for different horizontal eye gaze angle.

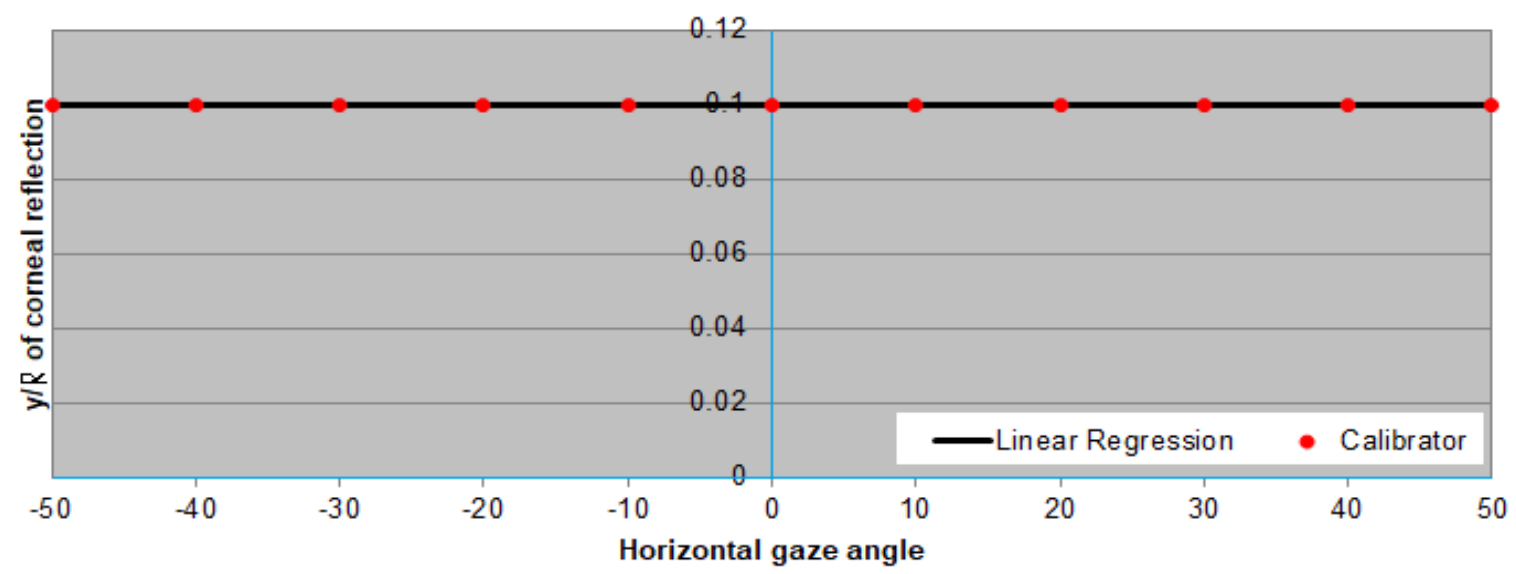

Figure 9. Horizontal gaze vs y/R 


\subsubsection{Vertical gaze estimation using corneal reflection}

Similar experiments which are done for horizontal eye gaze estimation has been done for vertical eye gaze estimation also. To find the relation between vertical gaze angle and corneal reflection position, data is captured with eye gaze shifting in vertical direction from -30 degree to 30 degree and horizontal eye gaze kept constant. Following are the key observations from this experiment:

1. It is observed that when camera is aligned with the centre of both eyes and the gaze is at the centre of the fixed pattern then the corneal reflection centre overlaps with the centre of pupil. When the gaze shifts upwards from the centre of the pattern then the corneal reflection moves downwards from the pupil centre. And when the gaze shifts downwards from the centre of the pattern then the corneal reflection moves upwards from the pupil centre. The corneal reflection moves farther from the pupil centre when the gaze shifts more upwards or downwards from the centre of the pattern.

2. ' $y / R^{\prime}$ varies with vertical eye gaze as a linear equation. When ' $y / R$ ' is plotted against varying vertical eye gaze angles (with horizontal gaze angle kept constant) then the plot as shown in Figure 10 is obtained.

3. Vertical eye gaze can be estimated from the following eye coordinates: PupilCenter y, CornealReflectionCenter y, radius of iris.

4. Relation between $y / R$ and vertical gaze angle holds true with varying distance between the camera and face for a given person.

5. Relation between ' $y / R$ ' and vertical gaze angles measured at different horizontal gaze angle shows similar pattern (linear relation)

6. ' $y / R$ ' values collected from multiple people is mixed and plotted and the curve fitting error is found to be minimal. 7. With the shift in camera little up from the eye horizon there is a shift in curve from zero.

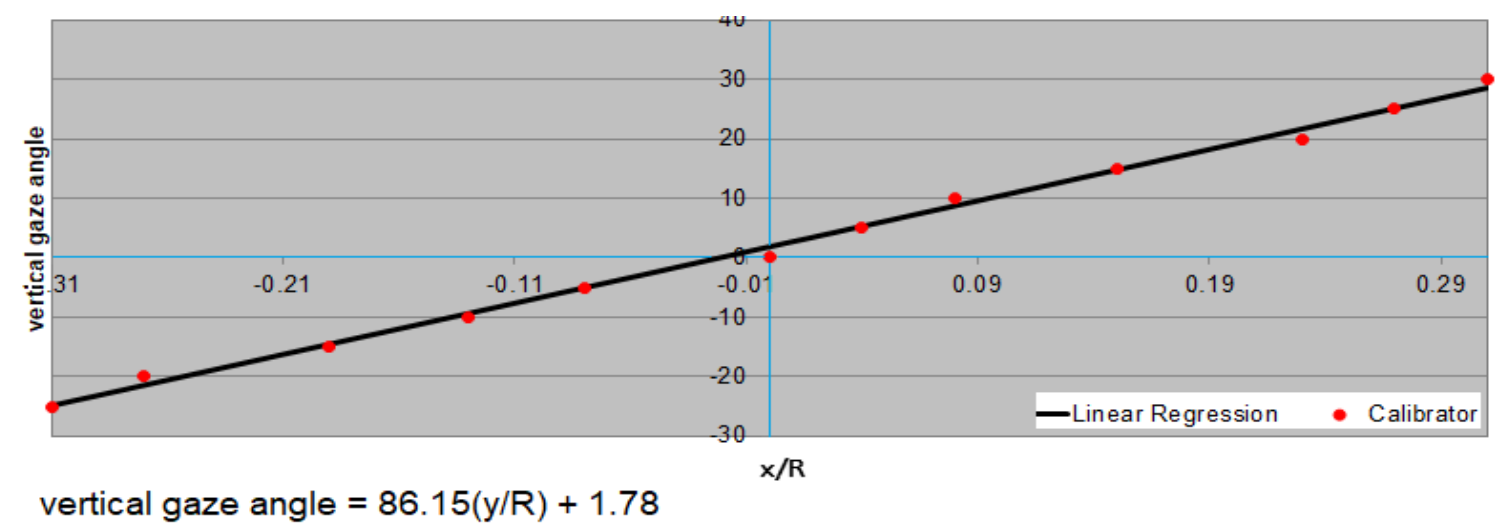

Figure 10. $y / R$ against vertical gaze change

\subsection{Pupil centre-based gaze estimation}

In this method the centre of pupil is tracked relative to the centre of eye for estimating gaze. For accurate and efficient comparison, the same set of images used for the corneal reflection 
movement analysis are used for pupil-based method as well. Measurements shown in 5 and 6 are used for gaze estimation using pupil centre position.

$$
\begin{aligned}
& \mathrm{x}=\mid \text { PupilCenter } \mathrm{x}-\text { EyeCenterx } \mid \\
& \mathrm{R}=\text { DistanceBetweenEyeCornerPoints } / 2
\end{aligned}
$$

\subsubsection{Horizontal gaze estimation using pupil centre}

To estimate horizontal gaze angle from pupil centre position, images of eye region are captured with gaze shifting horizontally from left to right with no head movement and the vertical gaze angle kept constant. The movement of pupil centre w.r.t the changes in gaze angle also shows some pattern but is different from the pattern formed by corneal reflection centre. When the gaze is at the centre of the pattern the pupil centre falls at the centre of eye. When the gaze shifts towards left or right the pupil centre moves in the same direction. This is unlike the corneal reflection position which moves in the opposite direction of gaze. But like corneal reflection movement, the movement of pupil centre with gaze change is not linear. To understand this nonlinear relation ' $\mathrm{x} / \mathrm{R}$ ' is plotted against horizontal gaze angle and the plot as shown in figure 12 is obtained. It can be observed from figure 12 that a third order polynomial is fitting the calibration points effectively.

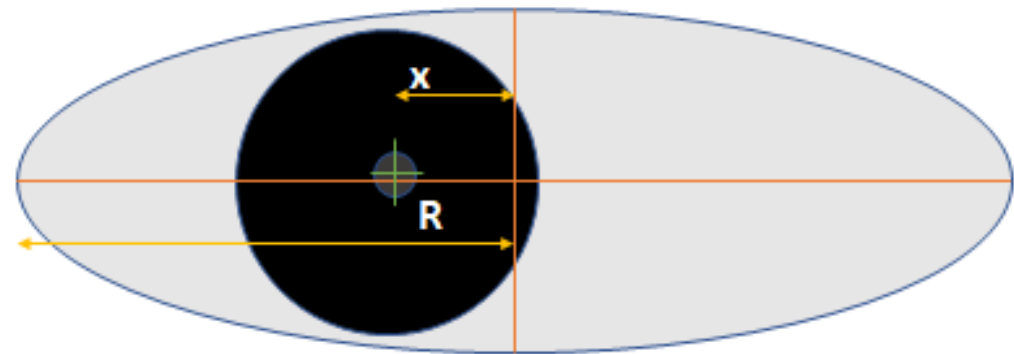

Figure 11. Measurements for gaze estimation from pupil centre

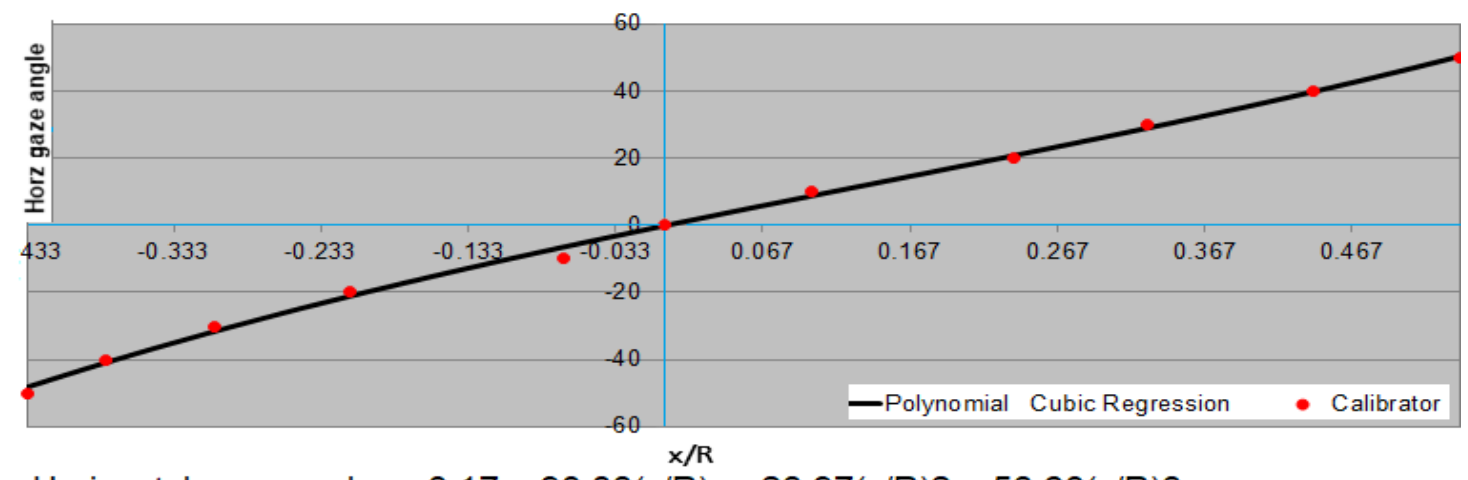

Horizontal gaze angle $=-0.17+90.83(x / R)+-23.97(x / R) 2+53.60(x / R) 3$

Figure 12. Pupil centre deviation with horizontal gaze change

From the polynomial curve it is clear that the horizontal gaze angle can be calculated from pupil centre position and width of eye. This relation between gaze angle and pupil position also holds true for varying distance between camera and eyes. This is also true at varying vertical eye gaze angle. Figure 13 has ' $\mathrm{x} / \mathrm{R}$ ' values at different vertical gaze angle and horizontal gaze angle equals to 30 degree. ' $\mathrm{x} / \mathrm{R}$ ' is a constant with varying vertical gaze angle. 


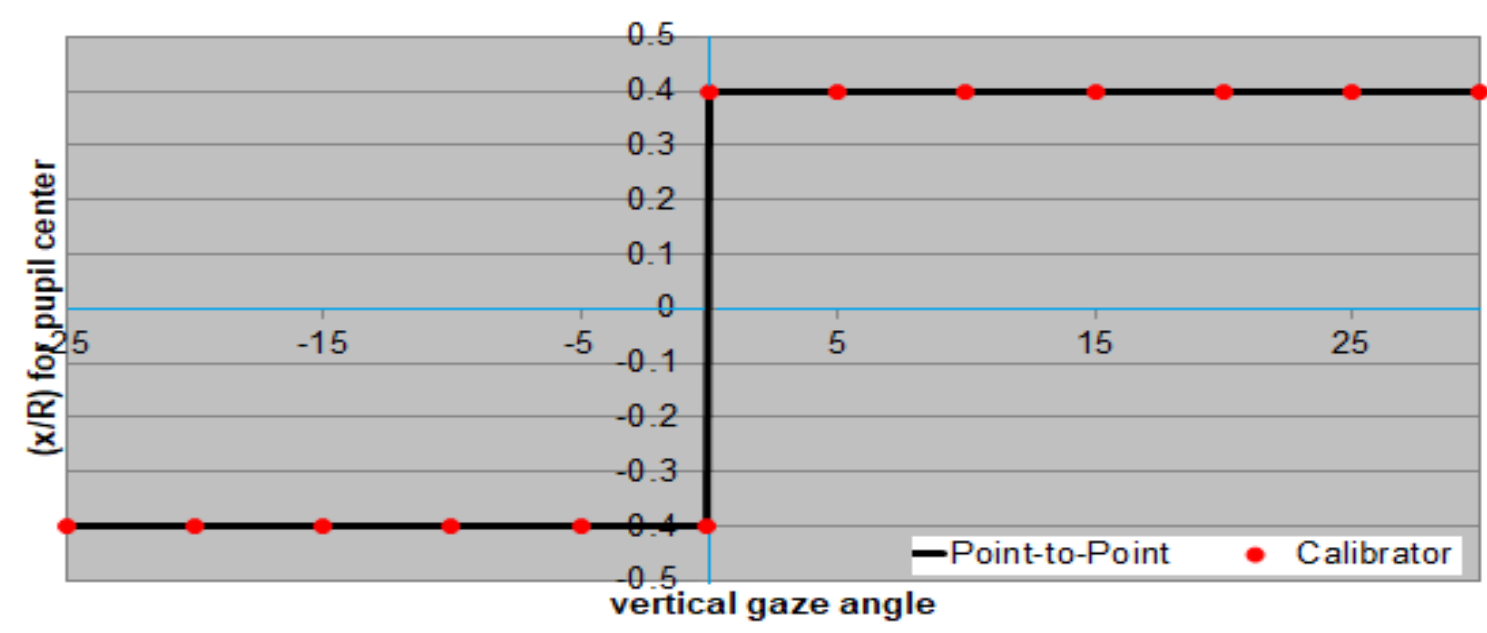

Figure 13. $\mathrm{x} / \mathrm{R}$ at varying vertical gaze angle

When the camera is not aligned with the centre of face, then there is a shift in the polynomial curve from zero.

To check how generic is pupil centre-based gaze estimation method ' $\mathrm{x} / \mathrm{R}$ ' values calculated from multiple people are mixed and a third order polynomial curve is fitted on these calibration points. Figure 14 has values from 3 different people marked in three different colours. The error in curve fitting is calculated and is found to be more when compared to the error obtained from the corneal reflection-based method. Table II has values of coefficients and its error values.

The polynomial curve in figure 14 is used to estimate gaze for person 1,2 and 3 and the error in gaze estimation is obtained as $3.72 \mathrm{deg}, 4.61 \mathrm{deg}$ and $4.88 \mathrm{deg}$ respectively. The higher error in the pupil-based approach is because of the variation in eye shape from person to person.

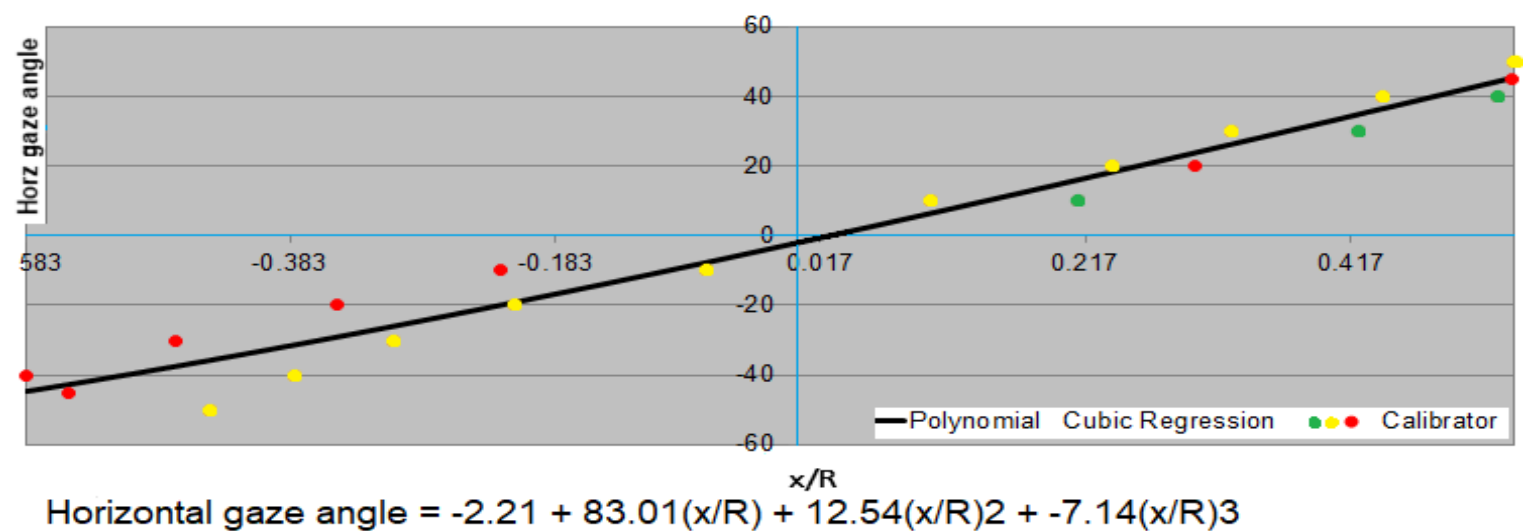

Figure 14. Pupil movement in different people 
Table 2: Error values of polynomial coefficients

\begin{tabular}{|c|c|c|}
\hline Coeff & Value & Error \\
\hline $\mathrm{a}$ & -2.21 & 2.67 \\
\hline $\mathrm{b}$ & 83.01 & 10.41 \\
\hline $\mathrm{c}$ & 12.54 & 14.43 \\
\hline $\mathrm{d}$ & -7.14 & 43.20 \\
\hline
\end{tabular}

\subsubsection{Vertical gaze estimation using pupil centre:}

To estimate vertical gaze angle from pupil centre position, images of eye region are captured with gaze shifting vertically and the horizontal gaze angle kept constant. Figure 15 is for vertical gaze angle equals $0,+10$ and -10 degree and horizontal gaze angle kept as 'zero'. In all the three cases the pupil centre is lying above the horizontal line connecting the eye corner points.

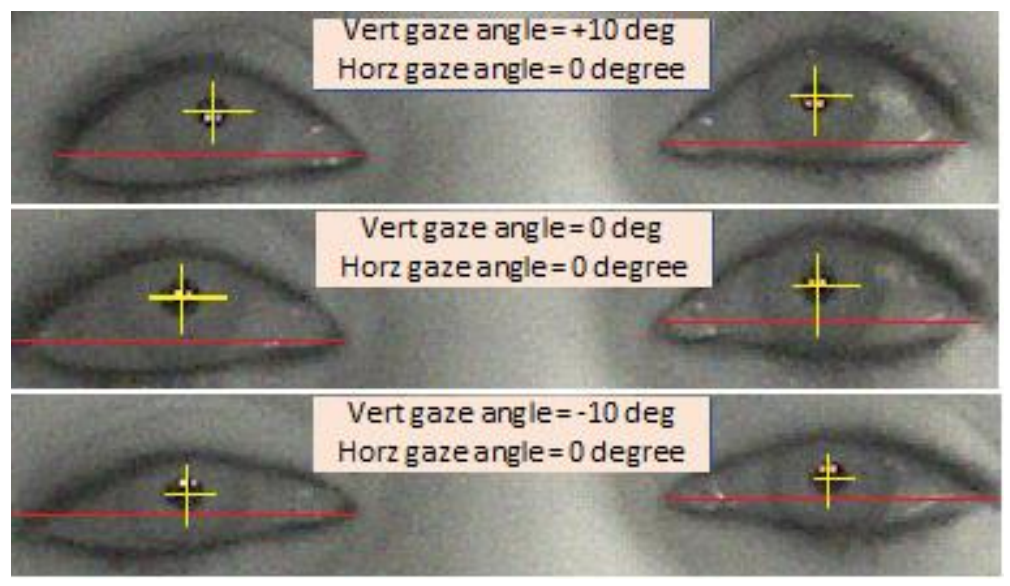

Figure 15. Pupil movement with vertical gaze change

In the case of Corneal reflection-based method, the target point is moving around the centre of pupil in a defined pattern and the pupil centre point can be considered as a reference point. All calculations to estimate eye gaze is independent of the width/height of the eye.

Whereas in the case of pupil centre-based method there is no fixed reference to estimate vertical gaze angle and hence the estimation becomes difficult. The variation in eye shape from person to person makes the gaze estimation challenging with pupil- based method.

\section{Minimum Gaze Angle Measurable}

In certain applications it is important to correctly differentiate the gaze change while the eye gaze is shifting between two points which are lying really closer. For example, to predict at which point on the infotainment cluster the driver is looking at, high precision gaze estimation techniques are required. The difficulty in gaze estimation increases when the need for precise and high-resolution gaze estimation is required. A comparative study is done on similar lines for pupil based and corneal reflection-based gaze estimation to find out which method is more efficient for precise high-resolution gaze estimation. In order to perform this comparison a fixed pattern in which the points are closely marked is placed in front of the person at $50 \mathrm{~cm}$ distance from eyes and the gaze is varied horizontally between these closely marked points from left to right. Images of resolution 1280 x 964 are captured using the IR camera. Deviation in corneal reflection centre 
from pupil centre and pupil centre from eye centre has been measured in pixels for each of the horizontal gaze angle. Table III contains the values, with the gaze changing from one point to next point with an offset of 1 degree.

Table 3: Minimum gaze angle measurable

\begin{tabular}{|c|c|c|}
\hline $\begin{array}{c}\text { Horz gaze } \\
\text { angle } \\
\text { (degree) }\end{array}$ & $\begin{array}{c}\text { CR } \\
\text { deviation } \\
\text { (pixels) }\end{array}$ & $\begin{array}{c}\text { Pupil } \\
\text { deviation } \\
\text { (pixels) }\end{array}$ \\
\hline 1 & 1 & 3 \\
\hline 2 & 2 & 3 \\
\hline 3 & 2 & 3 \\
\hline 4 & 2 & 4 \\
\hline 5 & 2 & 5 \\
\hline 6 & 3 & 5 \\
\hline 7 & 3 & 5 \\
\hline 8 & 3 & 7 \\
\hline 9 & 4 & 7 \\
\hline 10 & 4 & 8 \\
\hline 11 & 4 & 8 \\
\hline 12 & 5 & 9 \\
\hline 13 & 5 & 10 \\
\hline 14 & 5 & 10 \\
\hline 15 & 5 & 10 \\
\hline 16 & 5 & 11 \\
\hline 17 & 6 & 11 \\
\hline 18 & 6 & 11 \\
\hline 19 & 6 & 13 \\
\hline 20 & 6 & 13 \\
\hline
\end{tabular}

From the values shown in table III it can be inferred that the minimum gaze angle measurable with corneal reflection-based method is 4 degree and with pupil-based method is 3 degree for the given image resolution and distance from the camera. The resolution at which the gaze angle can be measured will always depend on the resolution of image captured. And for a given camera the resolution of gaze estimation depends on the distance of the camera from the eyes. With the increase in image resolution or with the camera placed more closer to the eyes, the gaze estimation resolution can be improved.

\section{Conclusions}

Gaze estimation method, its accuracy and efficiency are highly subjective to the use case it is meant for. Choosing the right method hence depends on many parameters. In this paper a comparative study of two methods are done under various scenarios. The pupil-based method has few limitations but it can work with any camera which can capture image of eye region clearly. Pupil based method doesn't need any IR illuminators and uses only eye features for gaze estimation. The corneal reflection method has few advantages, but it requires infra-red illuminators to illuminate the eyes for forming the bright spot on cornea. On the other hand, using IR camera and IR illuminators is useful in low light or dark scenarios like in driver monitoring systems. From the analysis done on the resolution of gaze estimation it is observed that pupil- 
based method has better gaze resolution than corneal reflection-based method with the given camera setup.

\section{ACKNOWLEDGEMENTS}

The authors would like to thank people who supported in data collection.

\section{REFERENCES}

[1] J. Sigut and S. Sidha, "Iris Center Corneal Reflection Method for Gaze Tracking Using Visible Light," in IEEE Transactions on Biomedical Engineering, vol. 58, no. 2, pp. 411-419, Feb. 2011.

[2] Sesma-Sanchez, Laura. (2016). Gaussian processes as an alternative to polynomial gaze estimation functions. 10.1145/2857491.2857509.

[3] Chennamma, Hr \& Yuan, Xiaohui. (2013). A Survey on Eye-Gaze Tracking Techniques. Indian Journal of Computer Science and Engineering. 4.

[4] Morimoto, Carlos\& Mimica, Marcio. (2005). Eye gaze tracking techniques for interactive applications. Computer Vision and Image Understanding. 98. 4-24. 10.1016/j.cviu.2004.07.010.

[5] D. W. Hansen, Q. Ji, "In the eye of the beholder: A survey of models for eyes and gaze", IEEE Trans. Pattern Anal. Mach. Intell., vol. 32, no. 3, pp. 478-500, Mar. 2010.

[6] Chunfei Ma, Seung-Jin Baek, Kang-A Choi, Sung-Jea Ko, "Improved remote gaze estimation using corneal reflection-adaptive geometric transforms," Opt. Eng. 53(5) 053112 (14 May 2014).

[7] Cazzato, D.; Leo, M.; Distante, C. An Investigation on the Feasibility of Uncalibrated and Unconstrained Gaze Tracking for Human Assistive Applications by Using Head Pose Estimation. Sensors 2014, 14, 8363- 8379.

[8] Gneo, M., Schmid, M., Conforto, S. et al. A free geometry modelindependent neural eye-gaze tracking system. J NeuroEngineering Rehabil 9, 82 (2012). https://doi.org/10.1186/1743-0003-9-82.

[9] Lin, Y., Lin, R., Lin, Y. et al. Real-time eye-gaze estimation using a low-resolution webcam. Multimed Tools Appl 65, 543-568 (2013).

[10] Ince, I.F., Kim, J.W. A 2D eye gaze estimation system with lowresolution webcam images. EURASIP J. Adv. Signal Process. 2011, 40 (2011).

[11] Wen Zhang, Tai-Ning Zhang, Sheng-Jiang Chang, "Eye gaze estimation from the elliptical features of one iris," Opt. Eng. 50(4) 047003 (1 April 2011).

[12] Brisson, J., Mainville, M., Mailloux, D. et al. Pupil diameter measurement errors as a function of gaze direction in corneal reflection eyetrackers. Behav Res 45, 1322-1331 (2013).

[13] M. S. Mounica, M. Manvita, C. Jyotsna and J. Amudha, "Low Cost Eye Gaze Tracker Using Web Camera," 2019 3rd International Conference on Computing Methodologies and Communication (ICCMC), Erode, India, 2019, pp. 79-85, doi: 10.1109/ICCMC.2019.8819645.

[14] Brunyé, T.T., Drew, T., Weaver, D.L. et al, "A review of eye tracking for understanding and improving diagnostic interpretation", Cogn. Research 4, 7 (2019).

[15] Ashraf, H., Sodergren, M. H., Merali, N., Mylonas, G., Singh, H., \& Darzi, A. (2018). Eye-tracking technology in medical education: a systematic review. Medical Teacher, 40(1), 62-69.

[16] A. Kar and P. Corcoran, "A Review and Analysis of Eye-Gaze Estimation Systems, Algorithms and Performance Evaluation Methods in Consumer Platforms," in IEEE Access, vol. 5, pp. 16495-16519, 2017, doi: 10.1109/ACCESS.2017.2735633.

[17] J. Lemley, A. Kar, A. Drimbarean and P. Corcoran, "Convolutional Neural Network Implementation for Eye-Gaze Estimation on Low-Quality Consumer Imaging Systems," in IEEE Transactions on Consumer Electronics, vol. 65, no. 2, pp. 179-187, May 2019, doi: 10.1109/TCE.2019.2899869. 


\section{AUTHORS}

Susmitha Mohan is a Lead engineer who has experience in developing various imaging and machine learning based solutions for automotive, aerospace and other domains. Worked in algorithm development from scratch, integrating components, testing and validating components as well as solutions, designing and modularization of algorithms. Worked closely with cross-functional teams like testing and validation, hardware porting, on road testing, data collection etc.

Manoj is an Imaging/Computer vision architect with wide experience in end to end product development including video/image processing, enhancement, view transformations, segmentation, object detection, recognition, tracking etc. Developed and managed solutions across multiple verticals like avionics, automotive, printing and document imaging, security and surveillance, manufacturing. Co-authored 3 USA patents granted for Automotive Driver Assistance Systems.
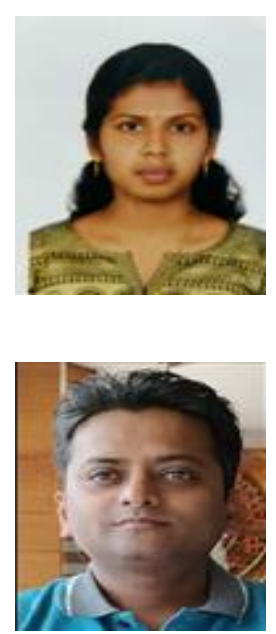

(C) 2020 By AIRCC Publishing Corporation. This article is published under the Creative Commons Attribution (CC BY) license 